\title{
PAPER
}

\section{Differences in the diagnostic accuracy of acute stroke clinical subtypes defined by multimodal magnetic resonance imaging}

\section{S J Allder, A R Moody, A L Martel, P S Morgan, G S Delay, J R Gladman, G G Lennox}

See end of article for authors' affiliations

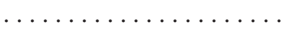

Correspondence to: Dr Steven J Allder, Department of Clinical Neurology, B floor, Medical School, Queen's Medical Centre,

Nottingham NG7 2RD UK; steve@

villaroad.freeserve.co.uk

Received 13 August 2002 In revised form

3 December 2002

Accepted

11 December 2002
Background: Despite its importance for acute stroke management, little is known about the underlying pathophysiology when patients with acute stroke are classified using clinical methods.

Objective: To examine the relation between the magnetic resonance defined stroke subtype and clinical stroke classifications using diffusion weighted imaging (DWI), perfusion weighted imaging (PWI), and angiographic magnetic resonance techniques.

Methods: Consecutive patients with clinical syndromes consistent with acute anterior circulation stroke were assessed clinically within six hours of onset and scanned as soon as possible using multimodal magnetic resonance imaging (MRI). Patients were classified clinically into total or partial anterior circulation syndromes using the Oxford classification, or according the severity of the National Institutes of Health stroke scale (NIHSS) (severe $>15$; mild/moderate $\leqslant 15$ ). At day seven, patients were classified by combining clinical course and MRI data as misdiagnosed, misclassified, suffering transient ischaemic attack, infarct with recanalisation, or infarction with persisting occlusion. Patients with occlusion were further divided on the basis of a large diffusion-perfusion mismatch.

Results: 84 patients with clinical anterior circulation syndromes were studied. Using the NIHSS, 42 were mild to moderate (0-15) and 42 were severe (> 15). There were 42 with partial anterior circulation syndromes (PACS) and 42 with total anterior circulation syndromes (TACS). Patients with TACS or severe stroke were more likely to have actually suffered a stroke (Fischer's exact test, $p=0.01$ ), to have a correctly classified stroke $\left(\chi^{2} 28.2, p<0.01\right)$, to have persisting occlusion $\left(\chi^{2} 30.6, p<0.01\right)$, and to have a large DWI-PWI mismatch $\left(\chi^{2} 17.1, p<0.01\right)$.

Conclusions: There is more inaccuracy in patients presenting with acute PACS or clinically mild to moderate anterior circulation stroke than in those with TACS or severe acute stroke syndromes. The latter appear more likely to be the targets for acute stroke interventions, as they include a significantly higher proportion of patients with persisting occlusion and diffusion/perfusion mismatch.
$\mathrm{T}$ here is a need for more accurate evaluation of patients presenting with acute stroke syndromes to enable treatment to be targeted with precision. ${ }^{1}$ New technologies such as diffusion weighted and perfusion weighted magnetic resonance imaging (MRI), xenon computed tomography (CT), single photon emission computed tomography (SPECT), and positron emission tomography (PET) may guide treatment in the future, but these will not become widely available for many years. ${ }^{2}$

The most widely available approach to the assessment of patients with acute ischaemic stroke is a combination of standard CT and clinical assessment. This has many potential limitations. Clinical means cannot distinguish between permanent and temporary impairments, nor identify the pathological type of stroke present. ${ }^{3}$ Brain CT is often normal in acute stroke despite later scans showing large areas of infarction, and routine CT does not provide information on cerebral perfusion or vascular anatomy. ${ }^{4}$ Thus it is common for a patient to present with a clinical deficit and to have normal brain CT. At that point the clinician does not know if the patient will prove to have a transient ischaemic attack (TIA), a minor stroke, or permanent disability, or even if death is likely.

The stroke syndrome is very heterogeneous but can be subdivided clinically using classifications based upon the presumed vascular territory involved, or upon the severity of the deficits. One of the most commonly used quasi-anatomical classifications is the Oxford classification, and one of the most commonly used measures of clinical deficit is the National Institutes of Health stroke scale (NIHSS). These are easy to apply and reliable. ${ }^{5}$ However, it is not known how closely they correlate with the underlying brain pathology during the first few hours of stroke when, presumably, effective acute treatments will need to be given.

We have studied a series of patients with anterior circulation syndromes to examine the relation between assessments based on the Oxford stroke classification or the NIHSS and the underlying pathology, as demonstrated by diffusion weighted imaging (DWI), perfusion weighted imaging (PWI), and magnetic resonance angiography (MRA).

\section{METHODS}

Consecutive patients presenting to our unit with syndromes consistent with anterior circulation stroke were recruited. The protocol was approved by our institution's ethics committee. All patients gave written consent where possible; patients' relatives were asked to give their written assent if the patient was unable to. All patients were examined within six hours of stroke onset by the same clinician (SJA), using a standardised neurological examination. These assessments were made before MRI and so were blinded to imaging data. Clinical syndromes were classified as either total or partial anterior circulation strokes (TACS, PACS), using the Oxford classification. Clinical deficits were measured using the NIHSS. Patients with NIHSS scores of $0-15$ have a good prognosis, whereas those with scores of $>15$ have a poor prognosis. We used this cut off to define "mild/moderate" and "severe" strokes. Patients with "severe" strokes on the NIHSS are considered to be equivalent to patients with TACS. 
Table 1 Baseline patient characteristics

\begin{tabular}{lllll}
\hline & Total & TACS & PACS & $\begin{array}{c}\text { p Value } \\
\text { (test) }\end{array}$ \\
\hline $\mathrm{n}$ & 84 & 42 & 42 & \\
Age (years) & 69 & 71 & 68 & $0.51(t$ test) \\
Sex (M/F) & $44 / 40$ & $20 / 22$ & $24 / 18$ & $0.34\left(\chi^{2}\right)$ \\
Diabetes (\%) & 14 & 11 & 17 & $0.17\left(\chi^{2}\right)$ \\
Hypertension (\%) & 37 & 40 & 34 & $0.24\left(\chi^{2}\right)$ \\
Atrial fibrillation (\%) & 18 & 19 & 17 & $0.42\left(\chi^{2}\right)$ \\
Ischaemic heart disease $(\%)$ & 16 & 19 & 13 & $0.27\left(\chi^{2}\right)$ \\
\hline F, female; M, male; PACS, partial anterior circulation syndrome; \\
TACS, total anterior circulation syndrome.
\end{tabular}

Patients were scanned as soon as possible after admission and all were scanned within 24 hours of the onset of symptoms. Time to imaging was defined as time from symptom onset. Patients were excluded if there was any change in their clinical NIHSS score before scanning. Patients were followed for the next seven days or until they were discharged.

For MRI we used a Siemens $1.5 \mathrm{~T}$ scanner (Erlangen, Germany) with an echoplanar booster for the diffusion weighted sequence. Standard T1 and T2 sequences were acquired to exclude coincidental disease and pre-existing infarction. The diffusion weighted sequence was a standard spin echo $(128 \times 128$ matrix, $1.95 \times 1.95 \times \times 5 \mathrm{~mm}$ voxel size, 18 slices, time of echo (TE) $123 \mathrm{~ms}, 0.8 \mathrm{~ms}$ per line, $\mathrm{b}=1100$ $\mathrm{s} / \mathrm{mm})$. Cerebral angiography of the circle of Willis was obtained using a two dimensional phase contrast technique (venc $=80 \mathrm{~cm} / \mathrm{s}$, time of repetition (TR) $80 \mathrm{~ms}$, TE $8.0 \mathrm{~ms}$, acquisitions $=5, \quad 1 \times 1 \times 35 \mathrm{~mm}$ voxel size). Perfusion weighted images were constructed using a dynamically acquired contrast enhanced technique that allowed depiction of all three arterial territories (anterior, middle, and posterior $)^{7}$ for a single slice.

One reader (ARM) visually assessed the images. He was blinded to the clinical status of the patient. Areas of high signal intensity on DWI were taken to indicate irreversible cerebral ischaemia. The phase contrast angiograms were used to determine whether the middle cerebral artery was patent or occluded. Perfusion weighted images were based on time to peak analysis of the contrast bolus transit.

On day 7, each patient's stroke was classified according to their initial scan and clinical state. The clinical state of the patient was determined by the stroke physician caring for the patients, who was blinded to the scan data. Patients were classified as misdiagnosed if initial DWI, PWI, and MRA were normal and an alternative clinical diagnosis became apparent. Patients with haemorrhage, small vessel occlusion (lacune), and posterior cerebral artery occlusion were identified on DWI and T2 imaging and assigned as misclassified. Clinical deficits in patients with transient ischaemic attacks had completely recovered within 24 hours; this diagnosis remained even if small areas of asymptomatic infarction were detected on DWI. Infarction with recanalisation was defined as evidence of persisting clinical signs, with acute cerebral infarction in the anterior circulation territory, and normal angiography and PWI. Infarction with persisting occlusion was defined when imaging revealed infarction with a persisting occlusion of the appropriate vessel on MRA. This group was further subdivided on the basis of whether or not there was a penumbra. Penumbra was defined as a mismatch in area of greater than $50 \%$ between the corresponding DWI and PWI slice. This was determined by visual assessment of the corresponding DWI and PWI images.

\section{Statistics}

We compared the proportion of patients in the clinically defined groups with each of the MR defined subgroups. All analysis was done using SPSS version 9. Subgroups were compared by $\chi^{2}$ analysis, with Fischer's exact test for expected cell sizes less than 5 .

\section{RESULTS}

Eighty four patients were entered into the study. No patient's clinical status changed between clinical assessment and imaging. All 84 patients were successfully imaged. The mean time to imaging was 11.4 hours. The mean NIH stroke score was 15.8 (range 6 to 23) and using this scale 42 were mild/moderate $(0-15)$ and 42 were severe $(>15)$. There were 42 with partial anterior circulation syndromes (PACS) and 42 with total anterior circulation syndromes (TACS). Three patients with TACS were classified as mild/moderate on the NIHSS, two patients had PACS and yet were classified as severe stroke using the NIHSS scale, and all other patients were similarly classified by both clinical assessment methods. Baseline characteristics are shown in table 1. There were no significant differences between the baseline characteristics of the mild/moderate and severe groups.

The results of the MRI classification are summarised in table 2. Of the seven patients with misdiagnosis, three had metabolic upset, one had a seizure, one had hemiplegic migraine, the symptoms were thought to be hysterical in one, and in one alcohol withdrawal was the final diagnosis. Of the 17 patients who were misclassified, eight had haemorrhage, six had small vessel occlusion (four in the brain stem, two in the internal capsule), and three had posterior circulation strokes. All seven patients who were initially misdiagnosed had PACS syndromes (Fischer's exact test, $p=0.01$ ). Most of those who were misclassified also had PACS rather than TACS syndromes $(16 / 17)\left(\chi^{2} 28.2, p<0.01\right)$. Seven of the 10 patients who proved to have a TIA initially had a TACS syndrome. Patients with TACS were significantly more likely to have an occlusion $\left(\chi^{2} 30.6, p<0.01\right)$ and a large DWI-PWI mismatch $\left(\chi^{2}\right.$ 17.1, $\left.\mathrm{p}<0.01\right)$. Similar differences were found between

Table 2 Results of multimodal magnetic resonance imaging classification by clinical subgroup

\begin{tabular}{lllllll}
\hline & & & & \multicolumn{2}{l}{ NIHSS } \\
\cline { 6 - 7 } & Total & TACS & PACS & $>15$ (severe) & 0-15 (mild/moderate) \\
\hline $\mathrm{n}$ & 84 & 42 & 42 & 42 & 42 \\
Misdiagnosis & 7 & 0 & 7 & 0 & 7 \\
Misclassification & 17 & 1 & 16 & 3 & 14 \\
TIA & 10 & 7 & 3 & 7 & 3 \\
Recanalised stroke & 15 & 4 & 11 & 5 & 10 \\
Occlusion with large mismatch & 20 & 16 & 4 & 15 & 5 \\
Occlusion without large mismatch & 15 & 14 & 1 & 12 & 3 \\
\hline
\end{tabular}

NIHSS, National Institutes of Health stroke scale score; PACS, partial anterior circulation syndrome; TACS, total anterior circulation syndrome; TIA, transient ischaemic attack. 
the mild/moderate and severe subgroups defined from the NIHSS. The differences in the number of patients misdiagnosed (Fischer's exact test, $\mathrm{p}=0.01)$ and misclassified $\left(\chi^{2}\right.$ $18.9, \mathrm{p}<0.01)$ between severe and mild/moderate subgroups were statistically significant. Additionally, patients with severe strokes had a higher proportion of persisting occlusions $\left(\chi^{2}\right.$ $17.9, \mathrm{p}<0.01)$ and a greater proportion of large DWI-PWI mismatches $\left(\chi^{2} 13.8, \mathrm{p}=0.01\right)$.

\section{DISCUSSION}

We have shown that patients with total anterior circulation syndromes or clinically severe scores on a stroke scale are more likely to be correctly diagnosed and classified than patients with partial anterior circulation deficits or those with milder clinical deficits. Furthermore the more severely affected groups are more likely to have a persisting occlusion and large a DWI-PWI mismatch than the patients with mild/moderate strokes.

The findings of this study are likely to be generalisable as the patients were prospectively and consecutively recruited from a routine clinical service. Use of a full standardised neurological examination by an interested physician should have maximised clinical accuracy. ${ }^{8}$ Employing the multimodal MRI sequences of DWI, PWI, and MRA allowed us to obtain a confident diagnosis and a precise classification in every patient. Potential difficulties with interpretation of these results could be introduced by the delay between clinical examination and imaging and by a relatively small sample size. However, no patient's clinical status changed between assessment and scanning, and the differences between groups are large.

Making direct comparisons with previous studies is difficult as the diagnostic accuracy of these two methods of classification have not previously been established in the very early phase of stroke. However, analysis of an alternative classification used in the TOAST trial (trial of ORG 10172 in acute stroke treatment) found a similar rate of error.' The diagnostic accuracy of the Oxford classification made at later time points has been extensively studied. ${ }^{10}$

Our results suggest that the total anterior circulation syndrome is pathophysiologically meaningful even when derived in the first six hours of stroke. By comparison the partial anterior circulation syndrome appears far less so. Partial anterior circulation syndromes have consistently been shown to have a lower sensitivity and specificity for accurately predicting infarct location than total anterior circulation syndromes, even when made at later time points. ${ }^{11}$ Difficulty with acute assessment may be related to the emergency environment in which the assessment is made and to the difficulty in correctly detecting subtle clinical signs in acutely ill or uncooperative patients. Although the total anterior circulation syndrome and the severity of the deficit proved to be more closely associated with the underlying pathophysiology than the partial anterior circulation syndrome or less severe deficits, it is important to note that those who proved to have TIA were in the severe group.

Mild/moderate patients were more likely to have a recanalised stroke and infrequently had a large DWI-PWI mismatch. By comparison, a large proportion of patients with severe stroke had persisting occlusion with a large mismatch. This finding is consistent with other studies. Patients with mild stroke syndromes have peripheral occlusive lesions which are presumably more liable to spontaneous recanalisation, and smaller volumes of brain tissue are at risk. By comparison, patients with large proximal occlusions show less spontaneous recanalisation, and large arterial territories are at risk. ${ }^{12}$ Additionally, previous MRI studies have shown that large DWI-PWI mismatches are associated with proximal occlusions. ${ }^{13}$

These findings have several important implications. Clinicians should be aware that there is considerable uncertainty over the diagnosis of a mild or partial anterior circulation stroke in the first few hours after the onset of symptoms. Furthermore, some of those in the more diagnostically robust total anterior circulation stroke or severe clinical deficit groups had TIA and therefore have little to gain from potentially hazardous acute interventions. Our findings illustrate the added value of detailed MRI studies in understanding what is happening to the brains of those presenting with acute stroke.

The close relation between a total anterior circulation syndrome, or a severe clinical deficit, and a PWI-DWI mismatch-which presumably represents the target for many stroke treatments-justifies the analysis of these subgroups in previously published stroke trials. These findings should also influence the selection criteria and power calculations of future intervention trials, and support the use of selection for future trials using MRI criteria where possible.

\section{ACKNOWLEDGEMENTS}

This study was supported by the Stroke Association (UK), the National Hospital Trust, and the Stanhope Neuroscience Trust. We would like to thank all the staff of the QMC MR centre and Professor Fentem for their support.

\section{Authors' affiliations}

S J Allder, G G Lennox, University Department of Clinical Neurology, Queen's Medical Centre, Nottingham, UK

A R Moody, P S Morgan, G S Delay, Academic Radiology, Queen's Medical Centre, Nottingham

A L Martel, Medical Physics, Queen's Medical Centre, Nottingham J R Gladman, Stroke Medicine and ADRU, Queen's Medical Centre, Nottingham

Competing interests: none declared

\section{REFERENCES}

1 Muir KW. Heterogeneity of stroke pathophysiology and neuroprotective clinical trial design. Stroke 2002;33:1545-50.

2 Heiss WD. Ischemic penumbra: evidence from functional imaging in man. J Cereb Blood Flow Metab 2000;20:1276-93.

3 Allder SJ, Moody AR, Martel S, et al. Limitations of clinical diagnosis in acute stroke [letter]. Lancet 1999;354:1523.

4 Bahn MM, Oser AB, DeWitte T. CT and MRI of stroke. J Magn Reson Imaging 1996;6:833-45.

5 Muir KW, Grosset DG. Neuroprotection for acute stroke: making clinical trials work. Stroke 1999;30:180-2.

6 Adams HP, Davis PH, Leira EC, et al. Baseline NIH stroke scale score strongly predicts outcome after stroke: a report of the trial of Org 10172 in acute stroke treatment (TOAST). Neurology 1999;53:126-31.

7 Moody AR, Martel A, Kenton A, et al. Contrast-reduced imaging of tissue concentration and arterial level (CRITICAL) for assessment of cerebral hemodynamics in acute stroke by magnetic resonance. Invest Radiol 2000;35:401-11.

8 Lodder J, Bamford J, Kappella J, et al. What causes false clinical prediction of small deep infarcts? Stroke 1994;25:86-91.

9 Madden KP, et al. Accuracy of initial stroke subtype diagnosis in the TOAST study. Trial of ORG 10172 in acute stroke treatment. Neurology 1995:45:1975-9

10 Stone SP, Allder SJ, Gladman JR. Predicting outcome in acute stroke. $\mathrm{Br}$ Med Bull 2000;56:486-94.

11 Mead GE, Lewis SC, Wardlaw P, et al. How well does the Oxfordshire community stroke project classification predict the site and size of the infarct on brain imaging? J Neurol Neurosurg Psychiatry 2000:68:558-62.

12 Bozzao L, Bastianello S, Fantozzi S, et al. Correlation of angiographic and sequential CT findings in patients with evolving cerebral infarction. Am J Neuroradiol 1989;10:1215-22.

13 Barber PA, Davis SM, Darby D, et al. Absent middle cerebral artery flow predicts the presence and evolution of the ischemic penumbra. Neurology 1999;52:1125-32. 